\title{
Deuterated methanol in the pre-stellar core L1544^
}

\author{
L. Bizzocchi ${ }^{1,2}$, P. Caselli ${ }^{2}$, S. Spezzano ${ }^{3}$, and E. Leonardo ${ }^{1}$ \\ ${ }^{1}$ Centro de Astronomia e Astrofísica, Observatório Astronómico de Lisboa, Tapada da Ajuda, 1349-018 Lisboa, Portugal \\ e-mail: [bizzocchi;elle]@oal.ul.pt \\ 2 Centre for Astrochemical Studies, Max-Planck-Institut für Extraterrestrische Physik, Gießenbachstraße 1, 85748 Garching, \\ Germany \\ e-mail: [caselli; bizzocchi]@mpe.mpg.de \\ 3 I.Physikalisches Institut, Universität zu Köln Zülpicherstraße 77, 50937 Köln, Germany \\ e-mail: spezzano@ph1.uni-koeln.de
}

Received 21 March 2014 / Accepted 21 July 2014

ABSTRACT

\begin{abstract}
Context. High methanol $\left(\mathrm{CH}_{3} \mathrm{OH}\right)$ deuteration has been revealed in Class 0 protostars with the detection of singly, doubly, and even triply D-substituted forms. Methanol is believed to form during the pre-collapse phase via gas-grain chemistry and then eventually injected into the gas when the heating produced by the newly formed protostar sublimates the grain mantles. The molecular deuterium fraction of the warm gas is thus a relic of the cold pre-stellar era and provides hints of the past history of the protostars.

Aims. Pre-stellar cores represent the preceding stages in the process of star formation. We aim at measuring methanol deuteration in L1544, a prototypical dense and cold core on the verge of gravitational collapse. The aim is to probe the deuterium fractionation process while the "frozen" molecular reservoir is accumulated onto dust grains.

Methods. Using the IRAM $30 \mathrm{~m}$ telescope, we mapped the methanol emission in the pre-stellar core L1544 and observed singly deuterated methanol $\left(\mathrm{CH}_{2} \mathrm{DOH}\right.$ and $\left.\mathrm{CH}_{3} \mathrm{OD}\right)$ towards the dust peak of L1544. Non-LTE radiative transfer modelling was performed on three $\mathrm{CH}_{3} \mathrm{OH}$ emissions lines at $96.7 \mathrm{GHz}$, using a Bonnor-Ebert sphere as a model for the source. We have also assumed a centrally decreasing abundance profile to take the molecule freeze-out in the inner core into account. The column density of $\mathrm{CH}_{2} \mathrm{DOH}$ was derived assuming LTE excitation and optically thin emission.

Results. The $\mathrm{CH}_{3} \mathrm{OH}$ emission has a highly asymmetric morphology, resembling a non-uniform ring surrounding the dust peak, where $\mathrm{CO}$ is mainly frozen onto dust grains. The observations provide an accurate measure of methanol deuteration in the cold pre-stellar gas. The derived abundance ratio is $\left[\mathrm{CH}_{2} \mathrm{DOH}\right] /\left[\mathrm{CH}_{3} \mathrm{OH}\right]=0.10 \pm 0.03$, which is significantly smaller than the ones found in lowmass Class 0 protostars and smaller than the deuterium fraction measured in other molecules towards L1544. The singly-deuterated form $\mathrm{CH}_{3} \mathrm{OD}$ was not detected at $3 \sigma$ sensitivity of $7 \mathrm{mK} \mathrm{km} \mathrm{s}^{-1}$, yielding a lower limit of $\left[\mathrm{CH}_{2} \mathrm{DOH}\right] /\left[\mathrm{CH}{ }_{3} \mathrm{OD}\right] \geq 10$, consistent with previous measurements towards Class 0 protostars.

Conclusions. The low deuterium fractionation observed in $\mathrm{L} 1544$ and the morphology of the $\mathrm{CH}_{3} \mathrm{OH}$ emission suggest that we are mainly tracing the outer parts of the core, where $\mathrm{CO}$ just started to freeze-out onto dust grains.
\end{abstract}

Key words. ISM: clouds - ISM: molecules - ISM: individual objects: L1544 - radio lines: ISM

\section{Introduction}

Deuterium is known to be $\sim 10^{5}$ times less abundant than hydrogen in the Universe (Linsky 2003); nonetheless, high abundances of D-containing isotopologues are common findings in many interstellar environments (e.g., Ceccarelli 2002; Roueff \& Gerin 2003). During the past couple of decades, observations have revealed large molecular deuteration in low-mass pre-stellar cores and Class 0 protostars, where singly, doubly, and - in some instances - triply deuterated molecules have been detected (see, e.g., Ceccarelli et al. 2007).

Methanol $\left(\mathrm{CH}_{3} \mathrm{OH}\right)$ is one of the species that show the highest D-enhancements: together with ammonia (Lis et al. 2002; van der Tak et al. 2002), it is one of the two molecules for which a triply deuterated form has been observed. $\mathrm{CD}_{3} \mathrm{OH}$ has been revealed in the low-mass protostar IRAS 16293-2422 (Parise et al. 2004), a source showing extreme deuterium enhancements. It is, however, not a pathological case, because Parise et al. (2006)

\footnotetext{
* Based on observations carried out with the IRAM $30 \mathrm{~m}$ Telescope. IRAM is supported by INSU/CNRS (France), MPG (Germany), and IGN (Spain).
}

also found very high abundances of $\mathrm{CH}_{2} \mathrm{DOH}$ and $\mathrm{CHD}_{2} \mathrm{OH}$ in an extended sample of low-mass Class 0 protostars.

Theoretical and experimental studies predict that methanol is formed on the grain surface by subsequent additions of hydrogen to iced CO (Tielens \& Hagen 1982; Watanabe \& Kouchi 2002). This process is thought to take place during the cold and dense pre-collapse phase (e.g., Öberg et al. 2011). Later on, the molecule is released in the gas when the heating of newly formed protostar sublimates the ice mantles (Ceccarelli et al. 2001). Methanol deuteration is thus likely to be produced completely by active grain-surface chemistry, controlled by the atomic D content of the accreting gas. The high atomic $\mathrm{D} / \mathrm{H}$ ratio required to account for the observed fractionation (0.1-0.2, Parise et al. 2002) has been explained by invoking an efficient transfer of atomic deuterium from the HD main reservoir via the intermediate $\mathrm{H}_{2} \mathrm{D}^{+} / \mathrm{D}_{2} \mathrm{H}^{+}$ions (Roberts et al. 2003; Parise et al. 2006), which are very abundant in the CO-depleted pre-stellar gas (e.g., Caselli et al. 2003; Phillips \& Vastel 2003; Vastel et al. 2004; Parise et al. 2011). Presently, all the measured methanol deuterations have been satisfactorily reproduced by the most recent coupled gas-grain models (Taquet et al. 2012; Aikawa et al. 2012), 
thus supporting the hypothesis that D-fractionation in methanol is a distinctive relic of the protostars' past history.

In this context, it is very interesting to study methanol deuteration in the pre-stellar gas. Starless cores represent the early stage of low-mass protostar evolution and offer the opportunity to probe the initial conditions in the process of star formation. These objects have a simple structure with no central heating source and little (thermal) turbulence, thus providing a favourable environment to study molecular deuteration. In particular, measuring deuterated methanol in pre-stellar cores yields a further test of the process responsible for the build-up of D-bearing isotopologue reservoir onto grain mantles.

A few studies of methanol in the pre-stellar gas have been reported so far. Earlier detections of the parent species were accomplished in TMC 1, TMC 1C, L134N (=L183), and B335 (Friberg et al. 1988; Takakuwa et al. 1998, 2000), and also in some translucent clouds by Turner (1998). Later on, $\mathrm{CH}_{3} \mathrm{OH}$ was also observed in L1498 and L1517B by Tafalla et al. (2006) and, together with its deuterated variant $\mathrm{CH}_{2} \mathrm{DOH}$, in the shocked gas of the Class 0 L1157 source (Codella et al. 2012). Previous detections of deuterated methanol in a sample of pre-stellar cores were also reported in a summarised form by Bacmann et al. (2007), but a detailed analysis of these observations is actually missing.

In this paper we report on the observation of $\mathrm{CH}_{2} \mathrm{DOH}$ towards the starless cloud L1544, thus providing an accurate assessment of the methanol deuteration in the cold pre-stellar gas. Multi-frequency analysis of the $\mathrm{CH}_{3} \mathrm{OH}$ emission (including non-LTE modelling) is performed in order to derive a reliable value for the column density of the main isotopologue. We finally compare the obtained fractionation ratio with the results derived in Class 0 protostars and discuss the implications suggested by the predictions based on gas-grain chemical models.

\section{Observations}

The methanol data presented here have been collected using the IRAM $30 \mathrm{~m}$ antenna, located at Pico Veleta (Spain) during three observing sessions in 2012-2013. Single-pointing observations towards the L1544 dust emission peak, located at coordinates $\alpha(\mathrm{J} 2000)=05^{\mathrm{h}} 04^{\mathrm{m}} 17.21^{\mathrm{s}}$ and $\delta(\mathrm{J} 2000)=+25^{\circ} 10^{\prime} 42.8^{\prime \prime}($ Caselli et al. 2002a), were carried out in October 2012, April 2013, and October 2013. The $3 \mathrm{~mm}$ lines of $\mathrm{CH}_{3} \mathrm{OH}$ were observed using several tunings of the EMIR E090 receiver while surveying various organic molecules in L1544 (Spezzano et al. 2013, and in prep.). We used the FTS backend in the "fine" configuration, resulting in $7.2 \mathrm{GHz}$ of instantaneous bandwidth divided in four sub-bands with a final unsmoothed resolution of $50 \mathrm{kHz}$. The $\mathrm{CH}_{2} \mathrm{DOH}$ transitions were only observed during the April 2013 observing block. The E090 and E150 receivers were tuned at 89.780 and $134.07 \mathrm{GHz}$, respectively, and the line signals were collected in the lower-inner (LI) sideband. As for $\mathrm{CH}_{3} \mathrm{OH}$, we used the FTS backend in the "fine" mode. Frequency-switching was adopted as observing mode using frequency throws of $3.9 \mathrm{MHz}$ at $3 \mathrm{~mm}$ and $7.8 \mathrm{MHz}$ at $2 \mathrm{~mm}$. The telescope pointing was checked every two hours on nearby bright radio quasars and was found accurate to 3-4". Typical system temperatures were $85-130 \mathrm{~K}$ at $97 \mathrm{GHz}$ and $170-220 \mathrm{~K}$ at 108 and $134 \mathrm{GHz}$. The observed spectra were then converted from the $T_{\mathrm{A}}^{*}$ to the $T_{\mathrm{mb}}$ temperature scale adopting $B_{\mathrm{eff}}$ and $F_{\text {eff }}$ values taken from the IRAM documentation. The rest frequencies and other spectroscopic parameters of the observed methanol lines are reported in Table 1.
Table 1. Spectroscopic parameters of the observed methanol lines.

\begin{tabular}{lcccc}
\hline \hline Line & $\begin{array}{c}\text { Rest frequency } \\
(\mathrm{MHz})\end{array}$ & $\begin{array}{c}E_{\mathrm{u}} / k_{b} \\
(\mathrm{~K})\end{array}$ & $g_{\mathrm{u}}{ }^{a}$ & $\begin{array}{c}A \\
\left(10^{-5} \mathrm{~s}^{-1}\right)\end{array}$ \\
\hline $\mathrm{CH}_{3} \mathrm{OH}$ & & & & \\
$2_{0,2}-1_{0,1}, A^{+}$ & 96741.375 & 6.96 & 5 & 0.3408 \\
$2_{1,2}-1_{1,1}, E_{2}$ & 96739.362 & $12.53^{b}$ & 5 & 0.2558 \\
$2_{0,2}-1_{0,1}, E_{1}$ & 96744.550 & $20.08^{b}$ & 5 & 0.3407 \\
$0_{0,0}-1_{1,1}, E_{1}-E_{2}$ & 108893.963 & $13.12^{b}$ & 1 & 1.471 \\
$\mathrm{CH}_{2} \mathrm{DOH}$ & & & & \\
$2_{0,2}-1_{0,1}, e_{0}$ & 89407.817 & 6.40 & 5 & 0.202 \\
$3_{0,3}-2_{0,2}, e_{0}$ & 134065.381 & 12.83 & 7 & 0.730 \\
$\mathrm{CH}_{3} \mathrm{OD}$ & & & & \\
$1_{1,0}-1_{0,1}, A^{-}-A^{+}$ & 133925.423 & 8.6 & 3 & 2.982 \\
\hline
\end{tabular}

Notes. Data are taken from $\mathrm{Xu} \&$ Lovas (1997) for $\mathrm{CH}_{3} \mathrm{OH}$, from Pearson et al. (2012) for $\mathrm{CH}_{2} \mathrm{DOH}$, and from Anderson et al. (1988) for $\mathrm{CH}_{3} \mathrm{OD} .{ }^{(a)}$ Rotational degeneracy. ${ }^{(b)}$ Energy relative to the ground $0_{0,0}$, $A$ rotational state.

L1544 was mapped during the Autumn 2013 session. We performed a $3^{\prime} \times 3^{\prime}$ on-the-fly (OTF) map centred on the source dust emission peak (see above). The reference position was set at $\left(-180^{\prime \prime}, 180^{\prime \prime}\right)$ offset with respect to the map centre. Three methanol lines at $96.7 \mathrm{GHz}$ were observed using two different E090 setups in the LI sideband and FTS in "fine" mode. The spectral axis was thus sampled with a $50 \mathrm{kHz}$ channel spacing. The map area was swept during $4.5 \mathrm{~h}$ of telescope time by moving the antenna along an orthogonal pattern of linear paths separated by 8 " intervals, corresponding to roughly one third of the beam FWHM (25.4" at 96.7 GHz). The mapping was carried out in good weather conditions $(\tau \sim 0.03)$ and a typical system temperature of $T_{\text {sys }} \approx 90 \mathrm{~K}$. The data processing was done using the GILDAS ${ }^{1}$ software (Pety 2005).

\section{Results}

\subsection{Mapping of $\mathrm{CH}_{3} \mathrm{OH}$}

Figure 1 shows the maps obtained for the $\mathrm{CH}_{3} \mathrm{OH} 2_{0,2}-1_{0,1}\left(A^{+}\right)$, $2_{1,2}-1_{1,1}\left(E_{2}\right)$, and $2_{0,2}-1_{0,1}\left(E_{1}\right)$ transitions. The last is appreciably weaker because it comes from a level of higher energy (see Table 1). The reference centre of the map is indicated and coincides with the maximum of the continuum $1.3 \mathrm{~mm}$ emission (Ward-Thompson et al. 1999).

In the right-hand panel of Fig. 2, we plot a grid of spectra of the $2_{0,2}-1_{0,1}\left(A^{+}\right)$and $2_{1,2}-1_{1,1}\left(E_{2}\right) \mathrm{CH}_{3} \mathrm{OH}$ lines. They were taken in nine positions separated by $30^{\prime \prime}$, as shown in the left-hand panel of the figure. Here, the grey scale represents the summed integrated intensity of the two lines, whereas the blue contours plot the $1.3 \mathrm{~mm}$ continuum emission map (Ward-Thompson et al. 1999) smoothed at 22". The lines peak strongly at the position $6,\left(0^{\prime \prime}, 30^{\prime \prime}\right)$, whereas the corresponding signals are reduced by one-half at the position 5 , which coincides with the map reference centre and is also very close to the maximum of the dust emission.

The $\mathrm{CH}_{3} \mathrm{OH}$ emission thus differs from the dust continuum and presents a single peak offset to the north-east. A weak intensity enhancement located to the south-west is also discernible, thus suggesting the presence of a highly asymmetrical broken ring distribution and reflecting the presence of a central

\footnotetext{
1 See GILDAS home page at the URL: http://www.iram.fr/ IRAMFR/GILDAS.
} 

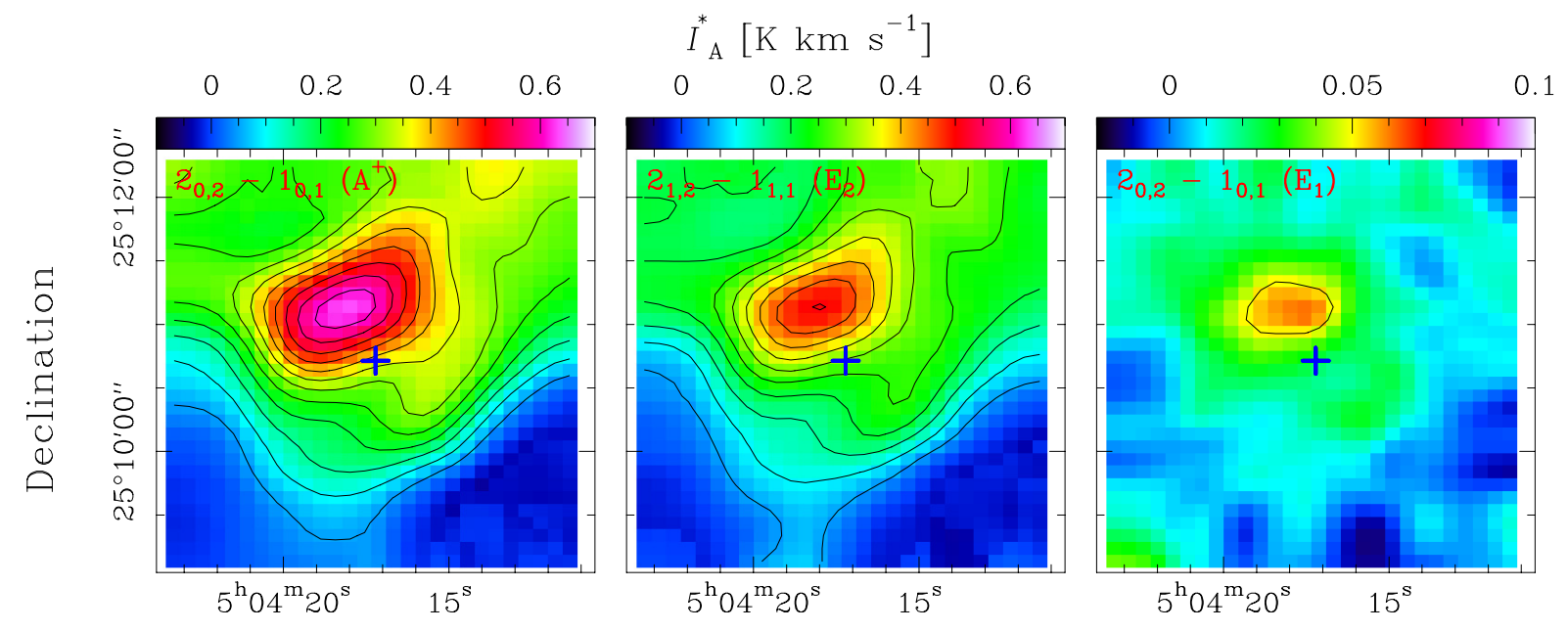

\section{Right ascension}

Fig. 1. Intensity maps (units of $T_{\mathrm{A}}^{*}$ ) of the $2_{0,2}-1_{0,1}\left(A^{+}\right), 2_{1,2}-1_{1,1}\left(E_{2}\right)$, and $2_{0,2}-1_{0,1}\left(E_{1}\right)$ transitions of $\mathrm{CH}_{3} \mathrm{OH}$ integrated over $0.5 \mathrm{~km} \mathrm{~s}{ }^{-1}$ velocity interval. The L1544 dust peak position located at $\alpha(\mathrm{J} 2000)=05^{\mathrm{h}} 04^{\mathrm{m}} 17.21^{\mathrm{s}}, \delta(\mathrm{J} 2000)=+25^{\circ} 10^{\prime} 42.8^{\prime \prime}$ is indicated by the blue cross marker. The first contour is at $5 \sigma$ and the increment is $5 \sigma$ for all three maps; note however that the colour scale is different for the weak $2_{0,2}-1_{0,1}\left(E_{1}\right)$ line (right panel). The images were smoothed to a $30^{\prime \prime}$ angular resolution to increase the signal-to-noise ratio $\left(1 \sigma \approx 10^{-2} \mathrm{~K} \mathrm{~km} \mathrm{~s}^{-1}\right)$.
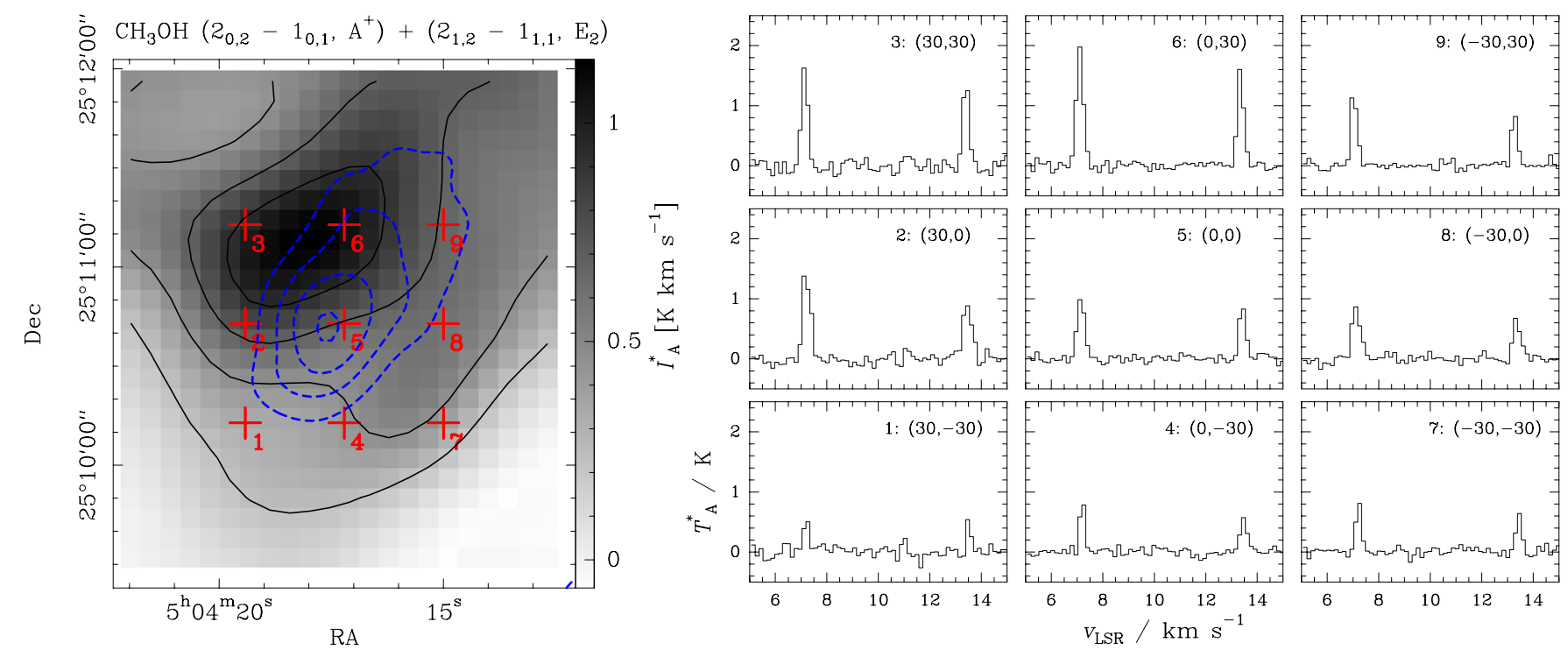

Fig. 2. Left panel: grey-scale map of the summed integrated intensity (units of $\left.T_{\mathrm{A}}^{*}\right)$ of the $2_{0,2}-1_{0,1}\left(A^{+}\right)$and $2_{1,2}-1_{1,1}\left(E_{2}\right) \mathrm{CH}_{3} \mathrm{OH}$ lines $\left(30^{\prime \prime}\right.$ angular resolution). Five equally spaced contours from 0.23 to $1.5 \mathrm{~K} \mathrm{~km} \mathrm{~s}^{-1}$ are plotted. The blue dashed contours plot the $1.3 \mathrm{~mm}$ continuum intensity map of Ward-Thompson et al. (1999) smoothed at 22" to improve the signal-to-noise ratio. Contours are at 100, 140, 180, and 220 mJy flux density levels. The red crosses represent the offset positions at which the spectra have been extracted. Right panel: map spectra of the $\mathrm{CH}_{3} \mathrm{OH}$ transitions towards the nine red crosses shown in the left panel. The velocity axis is centred on the $2_{0,2}-1_{0,1}\left(A^{+}\right)$line at $7.2 \mathrm{~km} \mathrm{~s}^{-1}$. The vertical axis of each spectrum represents the $T_{\mathrm{A}}^{*}$ scale in $\mathrm{K}$, as shown in the lower leftmost panel.

$\mathrm{CO}$ depletion hole. This feature is apparent in the $\mathrm{C}^{17} \mathrm{O}$ integrated intensity map shown in Caselli et al. (1999, see their Fig. 1). Also, the observed azimuthal asymmetry of the methanol emission is likely to be linked to slight inhomogeneities of the $\mathrm{CO}$ depletion, owing to the non-spherical and cometary-shaped morphology (e.g., Tafalla et al. 2004; Crapsi et al. 2007).

\section{2. $\mathrm{CH}_{3} \mathrm{OH}$ single-pointing observations}

Besides mapping, single-pointing, sensitive observations were performed towards the L1544 centre, corresponding to position 5 of Fig. 2 (dust emission peak). Four $\mathrm{CH}_{3} \mathrm{OH}$ emission lines were observed: three falling in a small frequency interval at $96.7 \mathrm{GHz}$ (the same mapped, see Fig. 1), plus one line at $108.9 \mathrm{GHz}$. The observations are shown in Fig. 3. Line profiles were analysed using the GAUSS task of CLASS and the resulting data are reported in Table 2 . The fit of the three closely spaced lines $2_{0,2}-1_{0,1}\left(A^{+}\right), 2_{1,2}-1_{1,1}\left(E_{2}\right)$, and $2_{0,2}-1_{0,1}\left(E_{1}\right)$ was carried out by adjusting only one "common" line width. Given the relatively small number of channels used in the least-squares fit, the statistical error on the optimised parameters yielded by the GAUSS procedure could be optimistic. Thus, to be on the safe side, we conservatively quote $3 \sigma$ uncertainties in Table 2.

The emitting levels have energies ranging from 7 to $20 \mathrm{~K}$, thus one may try to derive the $\mathrm{CH}_{3} \mathrm{OH}$ column density, $N$, and the average excitation temperature, $T_{\mathrm{ex}}$, through the population 

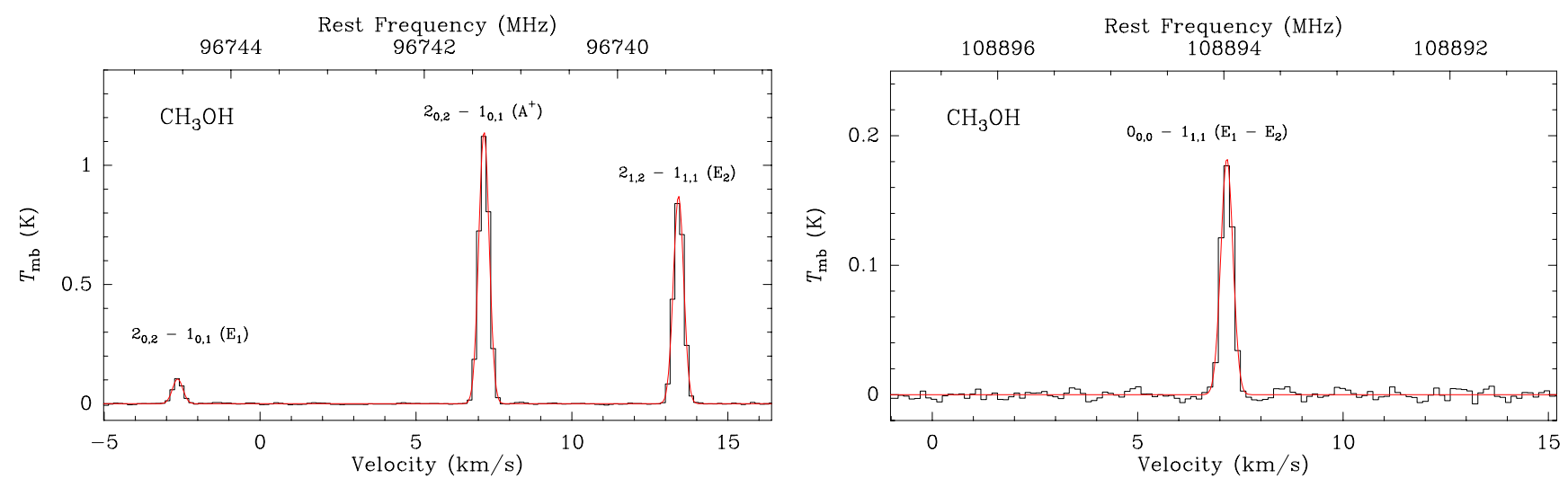

Fig. 3. $\mathrm{CH}_{3} \mathrm{OH}$ lines observed towards the $\left(0^{\prime \prime}, 0^{\prime \prime}\right)$ position of L1544. Left: group of $2_{1,2}-1_{1,1}$ and $2_{0,2}-2_{0,1}$ transitions. Right: $0_{0,0}-1_{1,1}\left(E_{1}-E_{2}\right)$ line. The spectral rms is $\sim 3 \mathrm{mK}$. The red solid line plots the Gaussian fit obtained using CLASS.

Table 2. Results of the CLASS GAUSS fit on the observed spectral profile of the methanol lines observed towards L1544.

\begin{tabular}{lccc}
\hline \hline Line & $\begin{array}{c}v_{\mathrm{LSR}} \\
\left(\mathrm{km} \mathrm{s}^{-1}\right)\end{array}$ & $\begin{array}{c}\int T_{\mathrm{A}}^{*} v \\
\left(\mathrm{mK} \mathrm{km} \mathrm{s}^{-1}\right)\end{array}$ & $\begin{array}{c}\Delta v \\
\left(\mathrm{~km} \mathrm{~s}^{-1}\right)\end{array}$ \\
\hline $\mathrm{CH}_{3} \mathrm{OH}$ & & & \\
$2_{0,2}-1_{0,1}\left(A^{+}\right)$ & $7.1804(11)$ & $472.4(25)$ & $0.3888(12)^{a}$ \\
$2_{1,2}-1_{1,1}\left(E_{2}\right)$ & $7.1808(14)$ & $360.7(25)$ & $0.3888(12)^{a}$ \\
$2_{0,2}-1_{0,1}\left(E_{1}\right)$ & $7.194(11)$ & $43.0(20)$ & $0.3888(12)^{a}$ \\
$0_{0,0}-1_{1,1}\left(E_{1}-E_{2}\right)$ & $7.1723(78)$ & $67.1(30)$ & $0.346(17)$ \\
$\mathrm{CH}_{2} \mathrm{DOH}$ & & & \\
$\left(2_{0,2}-1_{0,1}, e_{0}\right)$ & $6.889(24)$ & $30.5(42)$ & $0.358(60)$ \\
$\left(3_{0,3}-2_{0,2}, e_{0}\right)$ & $6.939(22)$ & $31.3(45)$ & $0.313(48)$ \\
\hline
\end{tabular}

Notes. Numbers in parentheses refer to $3 \sigma$ uncertainties in units of the last quoted digit. ${ }^{(a)}$ Fitted as average value.

diagram method (Goldsmith \& Langer 1999). We used here the modified method described by Nummelin et al. (2000), which also includes the cosmic background emission and the peak optical depth in addition to $T_{\text {ex }}$ and $N$. In this approach, the optimised parameters were sought by minimising the squared sum of the error weighted differences between observed and modelled line intensities. These intensities are derived through the radiative transfer equality

$T_{\mathrm{mb}}=\eta_{\mathrm{bf}}\left[J_{v}\left(T_{\mathrm{ex}}\right)-J_{v}\left(T_{\mathrm{bg}}\right)\right]\left(1-\mathrm{e}^{-\tau_{v}}\right)$,

where $\eta_{\mathrm{bf}}$ is the source-beam filling factor, and $J_{v}(T)$ is the equivalent Rayleigh-Jeans temperature. Assuming a Gaussian line profile, the peak opacity of each transition is obtained as

$\tau_{\nu}^{\text {(peak) }}=\sqrt{\frac{\ln 2}{16 \pi^{3}}} \frac{c^{3} A g_{\mathrm{u}} N}{v^{3} Q\left(T_{\mathrm{ex}}\right) \Delta v} \mathrm{e}^{-E_{\mathrm{u}} / k T_{\mathrm{ex}}}\left(\mathrm{e}^{h v / k T_{\mathrm{ex}}}-1\right)$.

Here, $v$ is the emission frequency, $\Delta v$ the FWHM line width in units of velocity, $E_{\mathrm{u}}$ the energy of the upper level (as listed in Table 1$), g_{\mathrm{u}}$ the rotational degeneracy, $A$ the Einstein's coefficient for spontaneous emission, and $Q(T)$ is the rotation partition function at temperature $T$. This is computed by summing over all $(A+E)$ rotational levels (Xu \& Lovas 1997), whose energies are available at the $\mathrm{CDMS}^{2}$ (Müller et al. 2005). Throughout the calculations, the average FWHM line width of $0.37 \mathrm{~km} \mathrm{~s}^{-1}$ was used and the beam filling factor $\eta_{\text {bf }}$ was set to unity.

\footnotetext{
2 Cologne Databases for Molecular Spectroscopy, URL: http:// www . astro.uni-koeln.de/cdms/
}

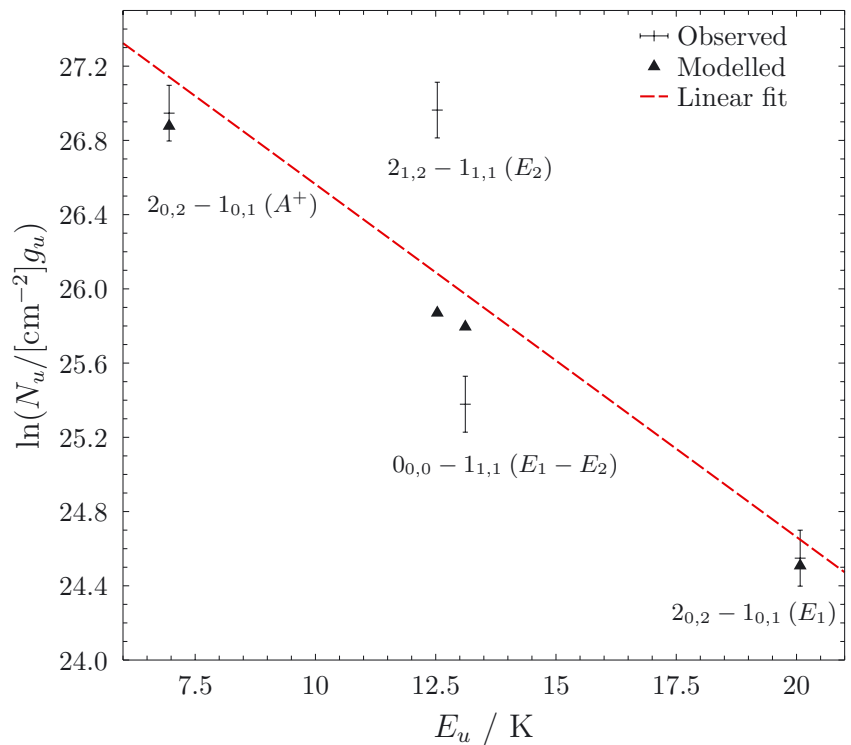

Fig. 4. Rotational diagram produced by the four $\mathrm{CH}_{3} \mathrm{OH}$ lines observed towards L1544. A considerable scatter is apparent. The red dashed line represent the "best" linear fit of all data and yields $T_{\mathrm{ex}}=6 \pm 3 \mathrm{~K}$ (see text).

Once the best-fit $N$ and $T_{\text {ex }}$ are determined through Eqs. (1) and (2), the match between observed and modelled data can be presented in a population diagram fashion, $\ln \left(N_{\mathrm{u}} / g_{\mathrm{u}}\right)$ vs. $E_{\mathrm{u}}$, where the $N_{\mathrm{u}}$ value for each transition is derived from the corresponding peak optical opacity through

$N_{\mathrm{u}}=\left[B_{v}\left(T_{\mathrm{ex}}\right)-B_{v}\left(T_{\mathrm{bg}}\right)\right] \sqrt{\frac{4 \pi^{3}}{\ln 2}} \frac{\tau_{v}^{\text {(peak) }} \Delta v}{h c A}$.

The result of this comparison is illustrated in Fig. 4. The observed points exhibit a considerable scatter, largely exceeding the estimated error bars $(\sim 15 \%$, including calibration and pointing uncertainties). The largest deviations are shown by the $2_{1,2}-1_{1,1}\left(E_{2}\right)$ line, which appears substantially brighter than expected. The best-fit modelled points instead lie on a straight line, not far from the linear fit obtained under the assumptions of optically thin emission and negligible background radiation (Goldsmith \& Langer 1999). Indeed, the derived peak optical depths are moderate $(\tau<0.4)$ and the cosmic background $\left(T_{\mathrm{bg}}=2.7 \mathrm{~K}\right)$ emission merely acts as an offset. Figure 4 clearly indicates that excitation anomalies are present, thus preventing determination of a unique excitation temperature for all 


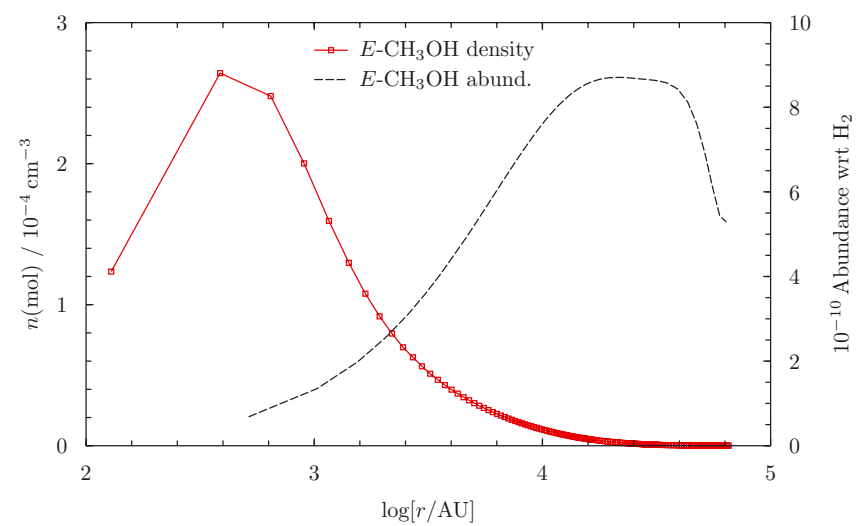

Fig. 5. Left panel: radial profiles for the $E-\mathrm{CH}_{3} \mathrm{OH}$ abundance (black) and density (red) corresponding to the best-fit input abundance of $9.2 \times$ $10^{-10}$. The radial trends were produced by the simple chemistry model implemented in the radiative transfer code MOLLIE (see text).

the rotational levels involved in the observed emissions. Indeed, the analysis yielded poorly constrained results: $T_{\mathrm{ex}}=6 \pm 3 \mathrm{~K}$ and a column density value $N=(1.9 \pm 1.9) \times 10^{13} \mathrm{~cm}^{-2}$.

\subsection{Non-LTE modelling}

To achieve a better constraint for the $\mathrm{CH}_{3} \mathrm{OH}$ column density in L1544, we performed a non-local thermodynamic equilibrium (LTE) modelling using the radiative transfer code MOLLIE (Keto \& Rybicki 2010) and the L1544 physical model with central density of $1 \times 10^{7} \mathrm{~cm}^{-3}$ described in Keto et al. (2014). The model is computed with a spherical Lagrangian hydrodynamic code with the gas temperature set by radiative equilibrium between heating by external starlight and cosmic rays and cooling by molecular line and dust radiation (see Keto \& Caselli 2008, for a comprehensive discussion of the theory). Even if the L1544 emission maps show that the cloud is not spherical but instead has an elongated shape, the adopted model is simple, physically motivated and certainly adequate to model observation data averaged over a single dish beam profile. For the statistical equilibrium calculation, we used de-excitation rates for $p$ $\mathrm{H}_{2} / A-\mathrm{CH}_{3} \mathrm{OH}$ and $p-\mathrm{H}_{2} / E-\mathrm{CH}_{3} \mathrm{OH}$ collisional systems (Rabli \& Flower 2010a) available at the LAMDA database (Schöier et al. 2005). Collisional data for $o-\mathrm{H}_{2}$ are lacking, but this does not represent a problem for our modelling because the $\mathrm{H}_{2}$ ortho-topara ratio (OPR) is expected to be very low in pre-stellar cores (see, e.g., Walmsley et al. 2004; Sipilä et al. 2013). Simulations were run separately for $A$ and $E$ symmetry species of methanol and considering only rotational levels below $36 \mathrm{~K}$.

MOLLIE implements a simple CO chemistry (Keto \& Caselli 2008) to take into account both the molecule freezeout towards the inner cloud core and the photo-dissociation due to the UV stellar field at the edges. Since CO is considered to be the main precursors of methanol, we used the same model to describe the molecular abundance trend across the core. The "nominal" $\mathrm{CH}_{3} \mathrm{OH}$ abundance, provided as the input parameter to MOLLIE, is thus internally translated in a radial abundance profile. As an example, the $E-\mathrm{CH}_{3} \mathrm{OH}$ radial abundance and density profile corresponding to the best-fit input abundance (see below) are illustrated in Fig. 5. The resulting beam-averaged value of the $\mathrm{CH}_{3} \mathrm{OH}$ column density (including both $A$ and $E$ species) is $2.66 \times 10^{13} \mathrm{~cm}^{-2}$.

At the end of the computation, the code outputs a data cube representing the spectral distribution of the emerging

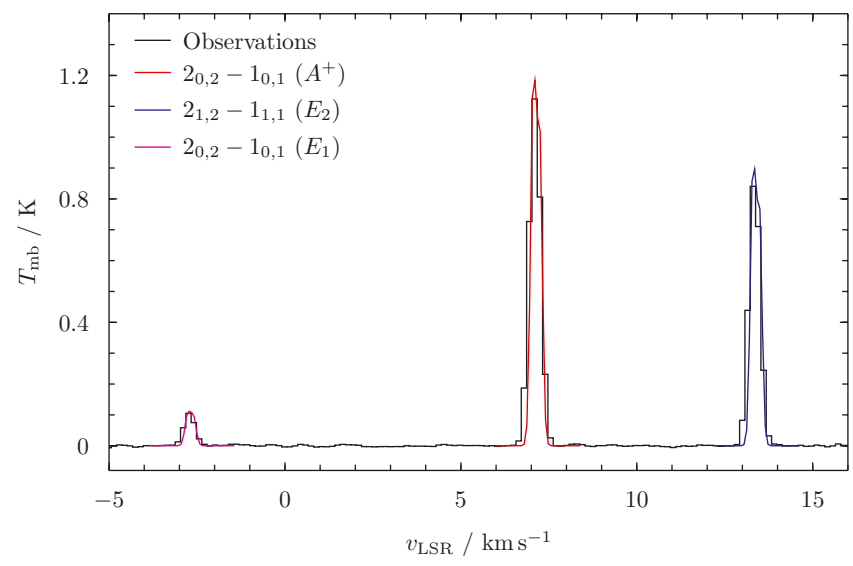

Fig. 6. Observed vs. modelled spectrum of $\mathrm{CH}_{3} \mathrm{OH}$ in $\mathrm{L} 1544$. The black histogram shows the observations. Coloured lines indicate the best-fit modelled spectra. The velocity axis is centred on the $2_{0,2}-1_{0,1}\left(A^{+}\right)$line at $7.2 \mathrm{~km} \mathrm{~s}^{-1}$.

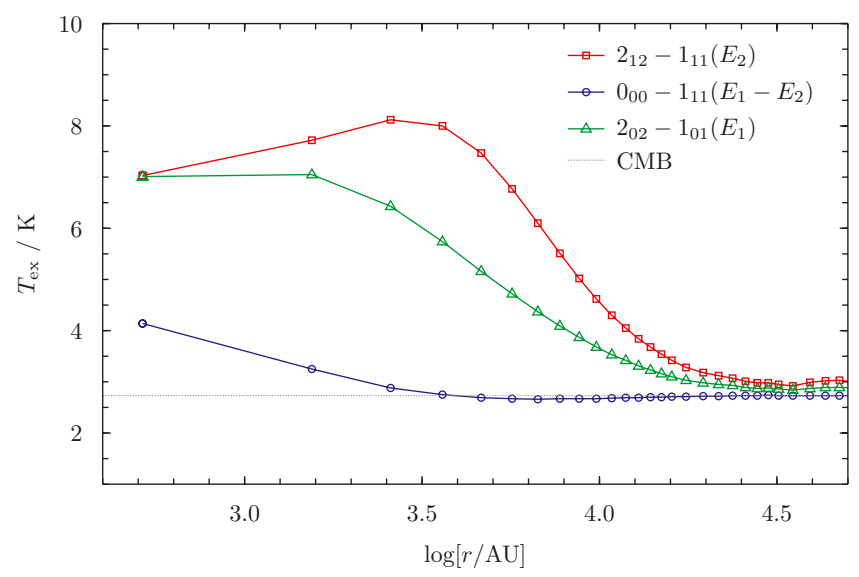

Fig. 7. Excitation temperature vs. cloud radius plot for the $2_{1,2}-1_{1,1}$, $2_{0,2}-1_{0,1}$, and $0_{0,0}-1_{1,1}$ transitions of $E-\mathrm{CH}_{3} \mathrm{OH}$ as computed by the present best-fit radiative transfer modelling.

radiation field. After convolution with the appropriate telescope beam $\left(25.5^{\prime \prime}\right.$ at $97.6 \mathrm{GHz}, 22.9^{\prime \prime}$ at $\left.108.9 \mathrm{GHz}\right)$, the modelled spectra were extracted from the central pixel and compared to the observations. Optimal $\chi^{2}$-fit between observed and modelled spectral profiles were found for the $2_{0,2}-1_{0,1}\left(A^{+}\right), 2_{1,2}-1_{1,1}\left(E_{2}\right)$, and $2_{0,2}-1_{0,1}\left(E_{1}\right)$ transitions using input abundances of $9.5 \times$ $10^{-10}$ and $9.2 \times 10^{-10}$, for $A$ and $E$ species, respectively. The modelling results for these three emissions falling at $96.7 \mathrm{GHz}$ are shown in Fig. 6.

Despite the success in reproducing these three lines, the fit underestimates the brightness of the observed $0_{0,0}-1_{1,1}\left(E_{1}-E_{2}\right)$ emission at $108.9 \mathrm{GHz}$ by a factor of 4 . Figure 7 illustrates the trend of the excitation temperatures for the modelled $E$ $\mathrm{CH}_{3} \mathrm{OH}$ transitions as a function of the cloud radius. It shows that the $0_{0,0}-1_{1,1}$ line is sub-thermally excited even at the high gas density of the cloud centre, where the other two $E$ lines are instead thermalised. The reason for this behaviour is easily understood by inspecting Fig. 8, which shows the bottom part of the rotational level diagram of $E-\mathrm{CH}_{3} \mathrm{OH}$, together with the radiative and collisional transitions considered in the present modelling. The $2_{0,2}$ and $2_{1,2}$ upper levels are connected to their corresponding lower state within the $K_{a}=0$ or $K_{a}=1$ manifolds by "strong" collisional transitions (i.e., upward rate $>10^{-11} \mathrm{~cm}^{3} \mathrm{~s}^{-1}$ at $10 \mathrm{~K}$ ), and other weaker $E_{1}-E_{2}$ connections also exist. On the other hand, the collisional transitions connecting $0_{0,0}$ to the 
A\&A 569, A27 (2014)

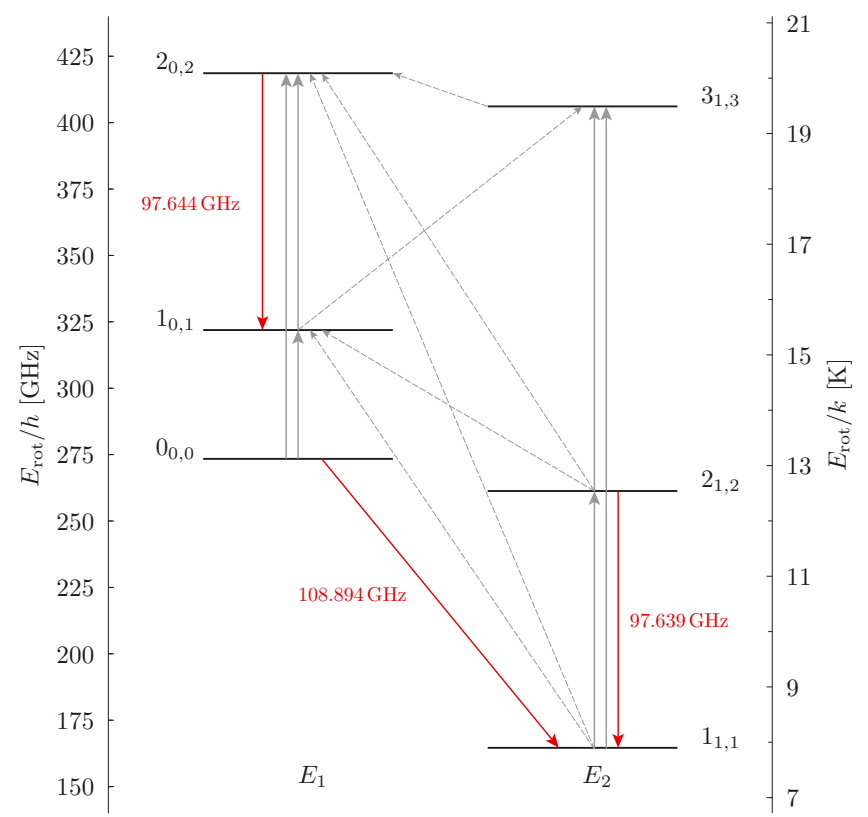

Fig. 8. Rotational energy plot for the lowest levels of $E-\mathrm{CH}_{3} \mathrm{OH}$. Active collisional channels are indicated by the upward grey arrows, with solid lines marking "strong" collisional transitions (upward rate $>10^{-11} \mathrm{~cm}^{3} \mathrm{~s}^{-1}$ at $10 \mathrm{~K}$, Rabli \& Flower 2010a). Red solid arrows indicate the observed radiative transitions.

lower $1_{1,1}$ and $2_{1,2}$ states have rate coefficients that are set to zero in the present $E-\mathrm{CH}_{3} \mathrm{OH} / p-\mathrm{H}_{2}$ data set (Rabli \& Flower 2010a). As discussed in Rabli \& Flower (2010b), this is an artefact of the coupled state (CS) approximation used in the production of the collision cross sections, and it is very likely that the collisional $0_{0,0}-1_{1,1}$ and $0_{0,0}-2_{1,2}$ transitions actually have small, but non-zero, rate coefficients.

Repeated tests using altered sets of collisional data showed that rate coefficients as small as $3 \times 10^{-11} \mathrm{~cm}^{3} \mathrm{~s}^{-1}$ ( $c a$. one third of the $2_{1,2}-1_{1,1}$ upward rate) allow for a satisfactory modelling of the $0_{0,0}-1_{1,1}\left(E_{1}-E_{2}\right)$ emission without significantly altering the fit quality of the $2_{1,2}-1_{1,1}\left(E_{2}\right)$ and $2_{0,2}-1_{0,1}\left(E_{1}\right)$ transitions. This finding thus suggests that the difficulties encountered in modelling the $0_{0,0}-1_{1,1}\left(E_{1}-E_{2}\right)$ line are indeed caused by small inaccuracies in the collisional dataset used.

An estimate of the error bar associated to the $\mathrm{CH}_{3} \mathrm{OH}$ column density was obtained as in Bizzocchi et al. (2013). The Gaussian width of the $\chi^{2}$ profile (plotted vs. the free parameter) is used to get an estimate of the uncertainty involved in the optimisation process $(\sim 19 \%)$. A further $10 \%$ error is added in quadrature to take the telescope calibration into account, yielding a final estimate of a $22 \%$ relative uncertainty. We thus ended with $N\left(\mathrm{CH}_{3} \mathrm{OH}\right)=(2.7 \pm 0.6) \times 10^{13} \mathrm{~cm}^{-2}$. This value is not far from the uncertain results derived using an LTE approach in Sect. 3.2, and it provides a more accurate constraint for the $\mathrm{CH}_{3} \mathrm{OH}$ column density in $\mathrm{L} 1544$.

\subsection{Singly deuterated methanol $\left(\mathrm{CH}_{2} \mathrm{DOH}\right)$}

Two lines of $\mathrm{CH}_{2} \mathrm{DOH}$ have been detected towards L1544, $2_{0,2}-1_{0,1}$ and $3_{0,3}-2_{0,2}$, at 98.4 and $134.1 \mathrm{GHz}$, respectively. Both are $a$-type transitions belonging to the lower $e_{0}$ torsional state (see Table 1). The observations are shown in Fig. 9, and the results of the CLASS Gaussian fits are reported in the last two rows of Table 2. Interestingly, both $\mathrm{CH}_{2} \mathrm{DOH}$ lines peak
Table 3. $\mathrm{CH}_{2} \mathrm{DOH}$ column densities determined in L1544 assuming optically thin emission and excitation temperature in the $5-8 \mathrm{~K}$ interval.

\begin{tabular}{lccc}
\hline \hline & \multicolumn{3}{c}{$N / 10^{12} \mathrm{~cm}^{-2}$} \\
\cline { 2 - 4 } & $T_{\mathrm{ex}}=5 \mathrm{~K}$ & $T_{\mathrm{ex}}=6.5 \mathrm{~K}$ & $T_{\mathrm{ex}}=8 \mathrm{~K}$ \\
\hline$Q(T)^{a}$ & 9.603 & 15.251 & 22.447 \\
$2_{0,2}-1_{0,1}\left(e_{0}\right)$ & 2.11 & 2.14 & 2.43 \\
$3_{0,3}-2_{0,2}\left(e_{0}\right)$ & 2.96 & 2.30 & 2.22 \\
\hline
\end{tabular}

Notes. ${ }^{(a)}$ Rotational partition function.

at a slightly bluer velocity compared to the ones of the parent species, which in turn are consistent with the systemic velocity of L1544 (7.2 $\mathrm{km} \mathrm{s}^{-1}$, Caselli et al. 2002a). The difference, $c a$. $0.3 \mathrm{~km} \mathrm{~s}^{-1}$, is small but significant given the fit statistical errors $\left(<0.003 \mathrm{~km} \mathrm{~s}^{-1}\right.$ for most lines) and the spectral channel spacing of the observations $\left(0.11-0.15 \mathrm{~km} \mathrm{~s}^{-1}\right)$. It is also unlikely to be due to uncertainties in the rest frequencies because they are predicted to be as small as $\sim 0.005 \mathrm{~km} \mathrm{~s}^{-1}$. The slight discrepancy is thus suggestive of a complex cloud dynamics, and it suggests that deuterated methanol traces a more confined (maybe inner) region with respect to its parent species. To confirm this, a map of $\mathrm{CH}_{2} \mathrm{DOH}$ is required.

The $\mathrm{CH}_{2} \mathrm{DOH}$ column density can be calculated from each transition assuming LTE conditions and optically thin emission once a suitable constraint for the excitation temperature, $T_{\mathrm{ex}}$, is available. It holds that

$N=\frac{8 \pi v^{3}}{c^{3}} \frac{Q\left(T_{\mathrm{ex}}\right)}{g_{\mathrm{u}} A_{\mathrm{ul}}} \frac{\mathrm{e}^{E_{\mathrm{u}} / k T_{\mathrm{ex}}}}{\mathrm{e}^{h v / k T_{\mathrm{ex}}}-1}\left[J_{v}\left(T_{\mathrm{ex}}\right)-J_{v}\left(T_{\mathrm{bg}}\right)\right]^{-1} \int T_{\mathrm{mb}} \mathrm{d} v$.

Given the uncertainty of the excitation temperature determined for the parent species, the column density of the deuterated variant is derived through Eq. (4) assuming $T_{\text {ex }}$ ranging between 5 and $8 \mathrm{~K}$. The results are collected in Table 3 .

The rotational partition function, $Q(T)$ (whose values are also reported in Table 3 ) refers to the entire $\mathrm{CH}_{2} \mathrm{DOH}$ population (i.e., it includes $e_{0}, e_{1}$, and $o_{1}$ levels) and was calculated by summing over rotational levels using the spectroscopic data of Pearson et al. (2012). The error bar of the $\mathrm{CH}_{2} \mathrm{DOH}$ column density is estimated adding in quadrature the calibration error $(\sim 15 \%)$ and the maximum dispersion due to the uncertainty in $T_{\text {ex }}$. In this way we obtain $N\left(\mathrm{CH}_{2} \mathrm{DOH}\right)=(2.4 \pm 0.9) \times$ $10^{12} \mathrm{~cm}^{-2}$.

We also performed sensitive observations at the frequency of the $1_{1,0}-1_{0,1}\left(A^{-}-A^{+}\right)$line of the singly deuterated $\mathrm{CH}_{3} \mathrm{OD}$ isotopologue, but they resulted in a non-detection. The achieved $3 \sigma$ sensitivity was $7 \mathrm{mK} \mathrm{km} \mathrm{s}^{-1}$ (assuming a line width similar to that found for $\mathrm{CH}_{2} \mathrm{DOH}$ ) thus, with $T_{\mathrm{ex}}$ constrained in the $5-8 \mathrm{~K}$ interval, we derived a $3 \sigma$ upper limit of $2.4 \times 10^{11} \mathrm{~cm}^{-2}$ for the beam-averaged $\mathrm{CH}_{3} \mathrm{OD}$ column density (see next section for a possible explanation of this non-detection).

\section{Discussion and conclusion}

We have detected two lines of $\mathrm{CH}_{2} \mathrm{DOH}$ in L1544 and carried out an accurate evaluation of the methanol deuteration in a cold pre-stellar gas. From the beam-averaged column densities computed in Sects. 3.3 and 3.4, we obtain a fractionation ratio $\left[\mathrm{CH}_{2} \mathrm{DOH}\right] /\left[\mathrm{CH}_{3} \mathrm{OH}\right]=0.10 \pm 0.03$, a value significantly lower than the ones measured in low-mass Class 0 protostars $(0.4-0.6$, 

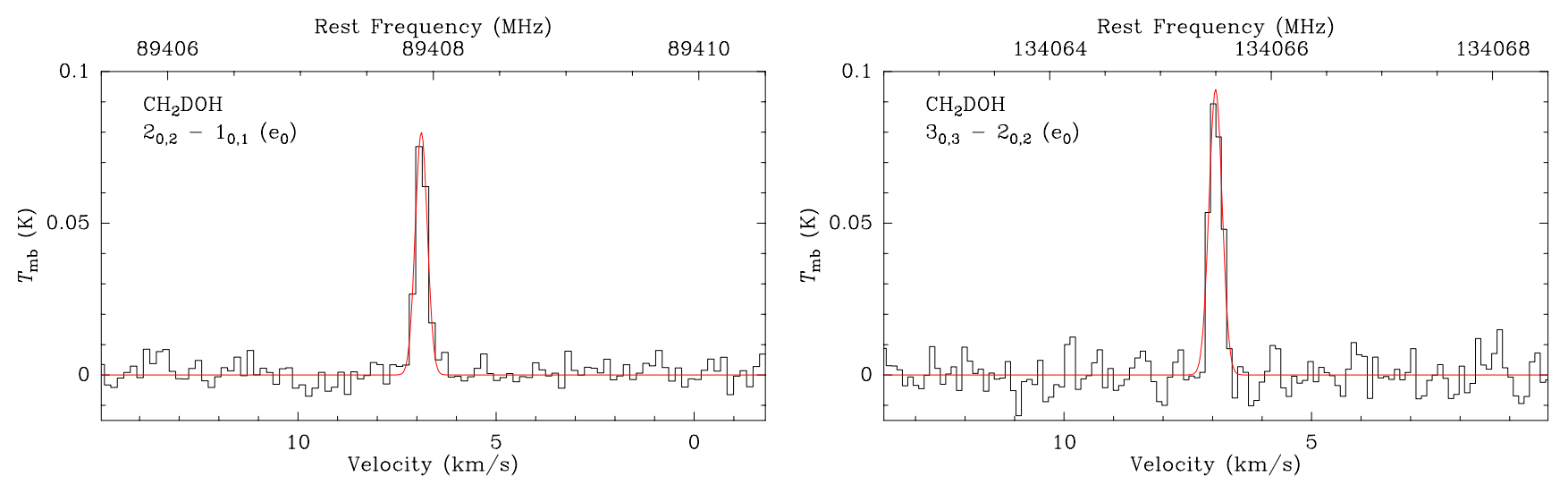

Fig. 9. $\mathrm{CH}_{2} \mathrm{DOH}$ lines observed towards the $\left(0^{\prime \prime}, 0^{\prime \prime}\right)$ position of L1544. Left: $2_{0,2}-1_{0,1}\left(e_{0}\right)-$ right: $3_{0,3}-2_{0,2}\left(e_{0}\right)$. The spectral rms is $\sim 5 \mathrm{mK}$. The red solid line plots the Gaussian fit obtained using CLASS.

Parise et al. 2006), and lower than the deuterium fraction measured in molecules such as $\mathrm{N}_{2} \mathrm{H}^{+}, \mathrm{NH}_{3}$, and $\mathrm{H}_{2} \mathrm{CO}$ (Caselli et al. 2002b; Bacmann et al. 2003; Roueff et al. 2005; Crapsi et al. 2007). However, it should be noted that, unlike methanol, all these molecules can also form in the gas phase, so that only our results reflect the surface chemistry activity directly.

State-of-the-art gas-grain chemical models of deuterium chemistry have been recently published by Aikawa et al. (2012) and Taquet et al. (2012). These studies follow the molecular evolution and the D-fractionation as star formation proceeds from the pre-collapse phase to a proto-stellar core. Both models are able to reproduce the high $\left[\mathrm{CH}_{2} \mathrm{DOH}\right] /\left[\mathrm{CH}_{3} \mathrm{OH}\right]$ ratio observed in Class 0 protostars if $\mathrm{D}$ and $\mathrm{H}$ abstraction and substitution are included in the chemical network. At densities of $5 \times 10^{6} \mathrm{~cm}^{-3}$ and $T=10 \mathrm{~K}$, the observed deuterium enhancement is reached in the molecular ice after $10^{4}-10^{5} \mathrm{yr}$ (relatively faster, $\sim 5 \times 10^{3} \mathrm{yr}$, following Taquet et al. calculations). On the other hand, a recent study of the gas-phase D-fractionation process (Kong et al. 2013) indicates that the gas within the inner core of L1544 has a deuteration age (estimated through $\mathrm{N}_{2} \mathrm{H}^{+}$) of five to eight times the free-fall time scale, which is of the order of $10^{4} \mathrm{yr}$. This suggests that the deuterium reservoir frozen on grains should be fully developed, at least in the central region of the core.

However, it should be noted that the column densities have been derived from observations towards the core centre, where $\mathrm{CH}_{3} \mathrm{OH}$ suffers a considerable depletion (see Fig. 1). Thus, the present observations may mostly be sensitive to the outer parts of the core, where the deuterium fractionation is lower than in the region traced by $\mathrm{N}_{2} \mathrm{H}^{+}$, a molecular ion not significantly depleted at high gas densities (e.g., Bizzocchi et al. 2013). The observed methanol deuteration is intermediate between the $\mathrm{N}_{2} \mathrm{D}^{+} / \mathrm{N}_{2} \mathrm{H}^{+}(0.2)$ and $\mathrm{DCO}^{+} / \mathrm{HCO}^{+}(0.04)$ found by Caselli et al. (2002b) in L1544. This gives the hint that methanol deuteration is indeed tracing the region where $\mathrm{CO}$ is freezing out (at densities of a few $10^{4} \mathrm{~cm}^{-3}$ ), where the $\mathrm{D} / \mathrm{H}$ ratio is not high enough to reach $\mathrm{D}$-fractions close to the ones found towards Class 0 sources or the centre of L1544. Inner regions are lost to view, since freeze-out is probably too efficient or because the product of deuteration remains on the grains, because centre grains are covered with $\mathrm{N}_{2}$ towards the core (see, e.g., Bertin et al. 2013). Also, the morphology of the $\mathrm{CH}_{2} \mathrm{DOH}$ emission in $\mathrm{L} 1544$ is not known, so the low measured $\left[\mathrm{CH}_{2} \mathrm{DOH}\right] /\left[\mathrm{CH}_{3} \mathrm{OH}\right]$ ratio might be produced by chemical inhomogeneities present in this pre-stellar core. Further progress in this study requires sensitive, interferometric observations aimed at deriving a detailed view of the fractionation in the different regions of the source.

From our non-LTE modelling results, we may infer that gasphase $\mathrm{CH}_{3} \mathrm{OH}$ in $\mathrm{L} 1544$ is composed of an almost equi-molar mixture of $A$ and $E$ species, and the resulting $[E] /[A]$ ratio is $0.97 \pm 0.26$. Given the large associated uncertainty, we may conclude that this value is consistent with the one implied by the picture of methanol formation on thermalised dust grains, (e.g., $[E] /[A] \approx 0.7$ at $10 \mathrm{~K})$.

The singly-deuterated $\mathrm{CH}_{3} \mathrm{OD}$ isotopologue was not detected by the present observations, yielding an upper limit of $2.4 \times 10^{11} \mathrm{~cm}^{-2}$ for its column density. The corresponding ratio between the singly-deuterated forms of methanol is $\left[\mathrm{CH}_{2} \mathrm{DOH}\right] /\left[\mathrm{CH}_{3} \mathrm{OD}\right] \geq 10$, higher than the nominal value of 3 that is predicted on a statistical basis assuming that $\mathrm{D}$ atoms are randomly distributed in the methanol isotopologues (Rodgers \& Charnley 2002). Our finding confirms the trend reported by Bacmann et al. (2007), and it agrees with the results of Class 0 protostars, where $\mathrm{CH}_{3} \mathrm{OD}$ appears to be underabundant with respect to the other methanol isotopic species $\left(\left[\mathrm{CH}_{2} \mathrm{DOH}\right] /\left[\mathrm{CH}_{3} \mathrm{OD}\right] \sim 14-20\right.$, Parise et al. 2006). Also, large $\left[\mathrm{CH}_{2} \mathrm{DOH}\right] /\left[\mathrm{CH}_{3} \mathrm{OD}\right]$ ratios have been found by Ratajczak et al. (2011) in a sample of low- to high-mass protostars.

The explanation of these "anomalous" ratios is still a challenge for the gas-grain chemical models, because $\mathrm{H}$ and D abstraction and substitution reactions - whose rates on ices are not very well constrained - are crucial to reproducing the observed abundances of the various methanol deuterated forms (e.g., Taquet et al. 2012). However, a laboratory study of lowtemperature formaldehyde hydrogenation (Hidaka et al. 2009) shows that the formation of $\mathrm{CH}_{2} \mathrm{DOH}$ in ice dominates $\mathrm{CH}_{3} \mathrm{OD}$, owing to the higher velocity of the H-D substitution process compared to the $\mathrm{D}$-atom addition.

The $\mathrm{CH}_{3} \mathrm{OH}$ emission has a highly asymmetric annular distribution surrounding the dust peak, where $\mathrm{CO}$ is mainly frozen onto dust grains. Methanol is expected to form via successive hydrogenation of $\mathrm{CO}$ on the surface of dust grains and then partially released in the gas phase upon formation (i.e., part of the formation energy is used to evaporate, in a process called reactive desorption; Garrod et al. 2006) and/or upon photo-desorption by UV photons produced by cosmic-ray impacts with $\mathrm{H}_{2}$ molecules (Prasad \& Tarafdar 1983). Evaporated methanol will then freeze-out onto dust grains in a time scale inversely proportional to the gas number density $\left(\sim 10^{9} / n_{\mathrm{H}} \mathrm{yr}\right.$; van Dishoeck et al. 1993). Thus, the gas phase abundance of methanol is expected to decrease towards the centre of the core, 
where the density (and the freeze-out rate) is higher and where the outer layers of ice mantles may be rich in $\mathrm{N}_{2}$, preventing hydrogenation of the underlying CO-rich layers (Bertin et al. 2013; Vasyunin \& Herbst 2013).

Acknowledgements. We are grateful to the IRAM $30 \mathrm{~m}$ staff for their support during the observations. L.B. and E.L. gratefully acknowledge support from the Science and Technology Foundation (FCT, Portugal) through the Fellowships SFRH/BPD/62966/2009 and SFRH/BPD/71278/2010. P.C. acknowledges the financial support of the European Research Council (ERC; project PALs 320620). L.B. also acknowledges travel support to Pico Veleta from TNA Radio Net project funded by the European Commission within the FP7 Programme.

\section{References}

Aikawa, Y., Wakelam, V., Hersant, F., Garrod, R. T., \& Herbst, E. 2012, ApJ, 760,40

Anderson, T., Crownover, R. L., Herbst, E., \& De Lucia, F. C. 1988, ApJS, 67, 135

Bacmann, A., Lefloch, B., Ceccarelli, C., et al. 2003, ApJ, 585, 55

Bacmann, A., Lefloch, B., Parise, B., Ceccarelli, C., \& Steinacker, J. 2007, in Molecules in Space and Laboratory (S. Diana)

Bertin, M., Fayolle, E. C., Romanzin, C., et al. 2013, ApJ, 779, 120

Bizzocchi, L., Caselli, P., Leonardo, E., \& Dore, L. 2013, A\&A, 555, A109

Caselli, P., Walmsley, C. M., Tafalla, M., Dore, L., \& Myers, P. C. 1999, ApJ, 523, L165

Caselli, P., Walmsley, C. M., Zucconi, A., et al. 2002a, ApJ, 565, 331

Caselli, P., Walmsley, C. M., Zucconi, A., et al. 2002b, ApJ, 565, 344

Caselli, P., van der Tak, F. F. S., Ceccarelli, C., \& Bacmann, A. 2003, A\&A, 403, L37

Ceccarelli, C. 2002, P\&SS, 50, 1267

Ceccarelli, C., Loinard, L., Castets, A., et al. 2001, A\&A, 372, 998

Ceccarelli, C., Caselli, P., Herbst, E., Tielens, A. G. G. M., \& Caux, E. 2007, Protostars and Planets V (Tucson: University of Arizona Press), 47

Codella, C., Ceccarelli, C., Lefloch, B., et al. 2012, ApJ, 757, L9

Crapsi, A., Caselli, P., Walmsley, M. C., \& Tafalla, M. 2007, A\&A, 470, 221

Friberg, P., Hjalmarson, A., Madden, S. C., \& Irvine, W. M. 1988, A\&A, 195, 281

Garrod, R., Park, I. H., Caselli, P., \& Herbst, E. 2006, Faraday Discuss., 133, 51

Goldsmith, P. F., \& Langer, W. D. 1999, ApJ, 517, 209

Hidaka, H., Watanabe, M., Kouchi, A., \& Watanabe, N. 2009, J. Phys. Conf. Ser., 194, 132024

Keto, E., \& Caselli, P. 2008, ApJ, 683, 238

Keto, E., \& Rybicki, G. 2010, ApJ, 716, 1315

Keto, E., Rawlings, J., \& Caselli, P. 2014, MNRAS, 440, 2616
Kong, S., Caselli, P., Tan, J. C., \& Wakelam, V. 2013, ApJ, submitted [arXiv: 1312.0971]

Linsky, J. L. 2003, Space Sci. Rev., 106, 49

Lis, D. C., Roueff, E., Gerin, M., et al. 2002, ApJ, 571, L55

Müller, H. S. P., Schlöder, F., Stutzki, J., \& Winnewisser, G. 2005, J. Mol. Spectrosc., 742, 215

Nummelin, A., Bergman, P., Hjalmarson, Å., et al. 2000, ApJS, 128, 213

Öberg, K. I., Boogert, A. C. A., Pontoppidan, K. M., et al. 2011, ApJ, 740, 109

Parise, B., Ceccarelli, C., Tielens, A. G. G. M., et al. 2002, A\&A, 393, L49

Parise, B., Castets, A., Herbst, E., et al. 2004, A\&A, 416, 159

Parise, B., Ceccarelli, C., Tielens, A. G. G. M., et al. 2006, A\&A, 453, 949

Parise, B., Belloche, A., Du, F., Güsten, R., \& Menten, K. M. 2011, A\&A, 526, A 31

Pearson, J. C., Yu, S., \& Drouin, B. J. 2012, J. Mol. Spectrosc., 280, 119

Pety, J. 2005, in EdP-Sciences Conference Series, eds. F. Casoli, T. Contini, J. Hameury, \& L. Pagani, SF2A-2005, 721

Phillips, T. G., \& Vastel, C. 2003, in SFChem 2002: Chemistry as a Diagnostic of Star Formation, eds. C. L. Curry, \& M. Fich, 3

Prasad, S. S., \& Tarafdar, S. P. 1983, ApJ, 267, 603

Rabli, D., \& Flower, D. R. 2010a, MNRAS, 406, 95

Rabli, D., \& Flower, D. R. 2010b, MNRAS, 403, 2033

Ratajczak, A., Taquet, V., Kahane, C., et al. 2011, A\&A, 528, L13

Roberts, H., Herbst, E., \& Millar, T. J. 2003, ApJ, 591, L41

Rodgers, S. D., \& Charnley, S. B. 2002, P\&SS, 50, 1125

Roueff, E., \& Gerin, M. 2003, Space Sci. Rev., 106, 61

Roueff, E., Lis, D. C., van der Tak, F. F. S., Gerin, M., \& Goldsmith, P. F. 2005 A\&A, 438, 585

Schöier, F. L., van der Tak, F. F. S., van Dishoeck, E. F., \& Black, J. H. 2005, A\&A, 432, 369

Sipilä, O., Caselli, P., \& Harju, J. 2013, A\&A, 554, A92

Spezzano, S., Brünken, S., Schilke, P., et al. 2013, ApJ, 769, L19

Tafalla, M., Myers, P. C., Caselli, P., \& Walmsley, C. M. 2004, A\&A, 416, 191

Tafalla, M., Santiago-García, J., Myers, P. C., et al. 2006, A\&A, 455, 577

Takakuwa, S., Mikami, H., \& Saito, M. 1998, ApJ, 501, 723

Takakuwa, S., Mikami, H., Saito, M., \& Hirano, N. 2000, ApJ, 542, 367

Taquet, V., Ceccarelli, C., \& Kahane, C. 2012, ApJ, 748, L3

Tielens, A. G. G. M., \& Hagen, W. 1982, A\&A, 114, 245

Turner, B. E. 1998, ApJ, 501, 731

van der Tak, F. F. S., Schilke, P., Müller, H. S. P., et al. 2002, A\&A, 388, L53

van Dishoeck, E. F., Blake, G. A., Draine, B. T., \& Lunine, J. I. 1993, in Protostar and planets III, ed. V. Mannings, A. P. Boss, \& S. S. Russell (University of Arizona Press), 163

Vastel, C., Phillips, T. G., \& Yoshida, H. 2004, ApJ, 606, L127

Vasyunin, A. I., \& Herbst, E. 2013, ApJ, 762, 86

Walmsley, C. M., Flower, D. R., \& Pineau des Forêts, G. 2004, A\&A, 418, 1035

Ward-Thompson, D., Motte, F., \& André, P. 1999, MNRAS, 305, 143

Watanabe, N., \& Kouchi, A. 2002, ApJ, 571, L173

Xu, L.-H., \& Lovas, F. J. 1997, J. Phys. Chem. Ref. Data, 26, 17 